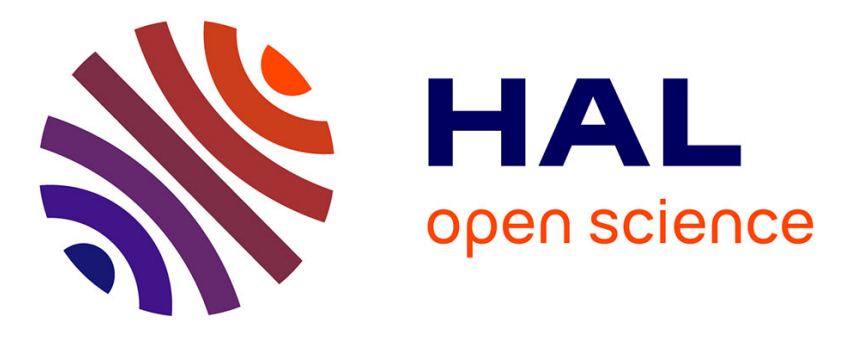

\title{
Technology Roadmap for Beyond 5G Wireless Connectivity in D-band
}

\author{
Jean-Baptiste Doré, Didier Belot, Eric Mercier, Simon Bicaïs, Gregory \\ Gougeon, Yoann Corre, Benoit Miscopein, Dimitri Kténas, Emilio Calvanese \\ Strinati
}

\section{To cite this version:}

Jean-Baptiste Doré, Didier Belot, Eric Mercier, Simon Bicaïs, Gregory Gougeon, et al.. Technology Roadmap for Beyond 5G Wireless Connectivity in D-band. 6G summit, Mar 2020, Levi, Finland. cea-02466751

\section{HAL Id: cea-02466751 https://hal-cea.archives-ouvertes.fr/cea-02466751}

Submitted on 4 Feb 2020

HAL is a multi-disciplinary open access archive for the deposit and dissemination of scientific research documents, whether they are published or not. The documents may come from teaching and research institutions in France or abroad, or from public or private research centers.
L'archive ouverte pluridisciplinaire HAL, est destinée au dépôt et à la diffusion de documents scientifiques de niveau recherche, publiés ou non, émanant des établissements d'enseignement et de recherche français ou étrangers, des laboratoires publics ou privés. 


\title{
Technology Roadmap for Beyond 5G Wireless Connectivity in D-band
}

\author{
Jean-Baptiste Doré ${ }^{1}$, Didier Belot ${ }^{1}$, Eric Mercier $^{1}$, Simon Bicaïs $^{1}$, Grégory Gougeon $^{2}$, Yoann Corre $^{2}$, \\ Benoit Miscopein ${ }^{1}$, Dimitri Kténas ${ }^{1}$, Emilio Calvanese Strinati ${ }^{1}$ \\ ${ }^{1}$ CEA-Leti, Minatec Campus, Grenoble, France - ${ }^{2}$ Siradel, Saint-Grégoire, France \\ contacts: \{jean-baptiste.dore, didier.belot, eric.mercier\}@cea.fr; ycorre@siradel.com
}

\begin{abstract}
Wireless communication in millimeter wave bands, namely above $20 \mathrm{GHz}$ and up to $300 \mathrm{GHz}$ is foreseen as a key enabler technology for the next generation of wireless systems. The huge available bandwidth is contemplated to achieve high data rate wireless communications, and hence, to fulfill the requirements of future wireless networks. Several Beyond 5G applications are considered for these systems: high capacity backhaul, enhanced hot-spot kiosk as well as short-range Deviceto-Device communications. In this paper we propose to discuss the trade-offs between scenario requirements and current silicon technologies limits to draw a technology roadmap for the next generation of wireless connectivity in D-band.

Index Terms-Millimeter wave communications, Integrated circuit technology, Radiofrequency integrated circuits
\end{abstract}

\section{INTRODUCTION}

Given their large unused spectrum, the sub-TeraHertz (sub$\mathrm{THz}$ ) frequency bands between 90 and $300 \mathrm{GHz}$ are potential candidates to achieve high-data rate wireless communications and hence to fulfill the requirements of future Beyond $5 \mathrm{G}$ (B5G) [1]. Considered applications include high capacity back-haul (100+ Gbps data rates) and enhanced hot-spot kiosk [2] [3]. Moreover, short range or Device-to-Device (D2D) communications could be deployed in sub- $\mathrm{THz}$ as this new spectrum would significantly increase transmission rates. As an example, an aggregated bandwidth of $58.6 \mathrm{GHz}$ was identified as possibly available for terrestrial radio-communications between 90 and $200 \mathrm{GHz}$. However before making this possible, many challenges need to be addressed. First of all, as the free-space propagation losses increase with the square of the frequency they have to be compensated by using highgain antennas, which entails severe constraints on antenna directivity and alignment. Besides, the connectivity link is mainly restricted to line-of-sight (LoS) or quasi-LoS situations, or requires a strong reflection. The design of antenna with electronically controlled steerable beam remains an open issue. Secondly, semiconductor technologies have to be chosen to meet expected performance at cost effective targets, including energy efficiency ones. The higher the frequency, the higher the price to pay [2] [4] [5] [6] .

in addition to challenges on analog/digital conversion, new physical (PHY) layer algorithms are required on the digital side to achieve high data-rate as traditional coherent schemes

The research leading to these results received funding from the French National Research Agency (ANR-17-CE25-0013) within the frame of the project BRAVE. do not consider the specific radiofrequency (RF) impairments related to the sub- $\mathrm{THz}$ bands. In particular, sub- $\mathrm{THz}$ systems could suffer from strong phase noise (PN) due to the poor performance of high-frequency oscillators [7]. Therefore the study of new digital transmission schemes, optimized to mitigate such impacts, like PN, is essential to guarantee good performance [8]. It should be noticed that an alternative PHY layer has been defined by the IEEE 802.15-3d [9] at the lower sub-THz frequency range between $252 \mathrm{GHz}$ and 325 $\mathrm{GHz}$ for switched Point-to-Point links. Many others solutions are currently under investigation to reach a trade-off between throughput, power consumption and complexity - see [10] as an example.

The contributions of this paper are the following. First, we review some potential scenarios in which the use of sub$\mathrm{THz}$ bands could give new opportunities. Then we analyze three applications by proposing dedicated PHY layers and link budgets based on propagation environment derived from a sub-THz ray-based model. Eventually, applications and requirements are cross-correlated with intrinsic silicon and III-V technologies features. This enables us to identify a technology roadmap fitting with next generation of wireless connectivity devices.

\section{CONTEMPlated SCENARIOS}

\section{A. System model}

For mmWave bands, system models have to be reconsidered w.r.t sub-6GHz band ones. The LoS direct-path is often the most dominant component. The propagation channel properties affect the link-level performance, therefore the service coverage. A fine characterization is required for realistic evaluation of new sub-THz behavior. Only few channel sounding campaigns, complex and costly, have been published yet [11] [12]. They are complemented by convenient numerical prediction, and produce on-demand channel models. In this work we use a model based on ray-tracing, extended up to the sub-THz frequencies [13]. This Volcano tool benefits from a LiDAR point cloud or detailed 3D representations to get a realistic prediction of the blockages and losses due to trees and street or indoor furnitures. The ray-tracing tool then predicts the propagation of both in-street back-haul or indoor mall scenario.

Regarding the electronic systems themselves, a critical impact from RF impairments, such as non-linearity, IQ imbal- 
ance and PN are expected. RF front-end analog blocks nonlinearities increase challenges in both modeling of circuits and anticipating the compensation measures. Power Amplifier (PA) efficiency and achievable output power are important targets to meet. In addition, the digitalization of wide bandwidths (BW) with power and integration constraints is still an open research topic. Last, PN, described by an uncorrelated Gaussian process for wide $\mathrm{BW}$ causes significant degradation in the communication performance.

Finding the optimum modulation scheme with regards to PN specifications has been largely investigated in the literature and remains a crucial topic to fulfil the requirement of future wireless technologies. Two different approaches can be proposed. First, envelop modulation, allowing non-coherent demodulation, could be considered. The information is carried by the signal amplitude and hence is inherently robust to severe phase impairments. Demodulation performance are far from the theoretical capacity but simple and energy optimized transceivers can be designed. In this case, maximizing the spectral efficiency per unit of energy is the optimization criterion. Second, when it comes to high spectral efficiency applications with band limited constraint, waveforms based on coherent modulations should be optimized to be robust to $\mathrm{PN}$. As a result, we have proposed in recent work the Polar Quadrature Amplitude Modulation (P-QAM) [8]. This modulation is defined as a rectangular lattice in the amplitudephase domain. The P-QAM balances coded information in the amplitude and phase components to meet constraints on PN robustness and Peak-to-Average Power Ratio (PAPR). Next sections of the paper will consider, for coherent demodulation, the P-QAM combined with 5GNR LDPC (Low Density Parity Check) FEC (Forward Error Correction) scheme for performance assessments.

\section{B. Back-haul outdoor scenario}

In this section, we give some figure of merits (FOM) of a Dband coherent transceiver deployed in an outdoor environment. First the performance of the PHY layer were first assessed to determine the best set of parameters: coding rate, modulation order and modulation shape given the SNR, the PN level and the targeted packet error rate (PER) of $10^{-2}$. Concerning the PHY layer, we assume a perfectly synchronized single carrier modulation. The input packet size of 1500 bytes is implemented with a coding rate ranging from 0.3 to 0.9 . Regarding numerical simulations, we further consider two PN levels: no PN $\sigma_{\phi}^{2}=0 \mathrm{rad}^{2}$ and strong $\sigma_{\phi}^{2}=10^{-1} \mathrm{rad}^{2}$. The strong PN levels corresponds to a noise floor spectral density $K_{0}$ of $-100 \mathrm{~dB}$ for a system bandwidth of $1 \mathrm{GHz}$. Resulting spectral efficiency goes from $0.6 \mathrm{bps} / \mathrm{Hz}$ at $-0.8 \mathrm{~dB} \mathrm{SNR}$, to $7.2 \mathrm{bps} / \mathrm{Hz}$ at $29.7 \mathrm{~dB}$ SNR without $\mathrm{PN}$ and $5.5 \mathrm{bps} / \mathrm{Hz}$ at $28.4 \mathrm{~dB}$ SNR for strong PN. The system is operated in the Dband, with a BW of possibly several tenths of $\mathrm{GHz}$, divided in $1 \mathrm{GHz}$ channels. The effective $\mathrm{BW}$ of the signal is $800 \mathrm{MHz}$ with a $20 \%$ overhead due to the control plane. The maximum reachable throughput is respectively 6.2 and $4.4 \mathrm{Gbps} / \mathrm{channel}$ when $\sigma_{\phi}^{2}=0$ and strong PN are considered.
TABLE I: Simulation parameters.

\begin{tabular}{|c|c|c|c|c|}
\hline Parameters & Notation & Outdoor & Indoor N2N & Indoor N2R \\
\hline Carrier frequency & $f_{c}$ & \multicolumn{3}{|c|}{$150 \mathrm{GHz}$} \\
\hline Channel bandwidth & $B$ & \multicolumn{3}{|c|}{$1 \mathrm{GHz}$} \\
\hline Allocated bandwidth & $B_{a}$ & \multicolumn{3}{|c|}{$0.8 \mathrm{GHz}$} \\
\hline Thermal noise & $N_{0}$ & \multicolumn{3}{|c|}{$-174 \mathrm{dBm} / \mathrm{Hz}$} \\
\hline Noise figure & $N_{f}$ & \multicolumn{3}{|c|}{$10 \mathrm{~dB}$} \\
\hline Transmit power & $P_{\mathrm{Tx}}$ & $30 \mathrm{dBm}$ & $19 \mathrm{dBm}$ & $19 \mathrm{dBm}$ \\
\hline Tx/Rx antenna gain & $\left(g_{\mathrm{Tx}}, g_{\mathrm{Rx}}\right)$ & $(25,25) \mathrm{dBi}$ & $(25,25) \mathrm{dBi}$ & $(25,8) \mathrm{dBi}$ \\
\hline Implementation loss & $L$ & \multicolumn{3}{|c|}{$2 \mathrm{~dB}$} \\
\hline Control plane overhead & $\mu$ & \multicolumn{3}{|c|}{20 p.c } \\
\hline
\end{tabular}

These numerical evaluations assume a city center environment while targeting a high capacity mesh back-haul application. The system performance is characterized for an outdoor mesh back-haul scenario, which could be exploited to feed an ultra-broadband mobile access network or fixed wireless access (FWA) network. The sub-THz devices are supposed to be installed on lampposts, located along the streets at 8 meters above the ground, in order to transport the data streams from a fiber PoP (Point of Presence) to dense local high-speed access points (AP) that either provide mobile or FWA typically at a lower frequency. The antenna beam is supposed to be perfectly aligned on the dominant propagation path, which is either the direct LoS path or a reflected path. Other transmission parameters are given in Table I. The propagation paths and losses are predicted from the estimated SNR which is mapped to the spectral efficiency. A channel bonding scheme could increase the aggregated throughput and allow to benefit from the available free spectrum offered in the sub-THz bands. The choice of a channelization of $1 \mathrm{GHz}$ is motivated by current state-of-the-art (SOTA) of analog-to-digital converters

The heat maps in Fig. 1 illustrates how the lamppost-tolamppost link performs depending on the distance, the propagation condition, the PN level and the selected modulation scheme. The illustrated environment is a square in the middle of a dense urban center with medium tree density. The peak throughput per channel is computed between two aligned sub$\mathrm{THz}$ devices; the first one at the center of the figure; the second one at any pixel of the map. Note that the actual lamppost locations, where this second device could precisely be positioned, are shown by black dots. The connectivity is strongly affected by building and foliage obstructions, while LoS propagation and strong clear reflections lead to the best performance. These throughput heat maps demonstrate that optimizing the modulation and demodulation schemes for PN channels results in significant performance gains for future sub-THz systems.

\section{Mesh indoor networks}

The optical fiber is the best solution to deliver strong capacity in the different blocks of a venue, large ones like e.g. airports, railway stations, stadium, campus, or malls, but a wireless mesh back-haul based on sub-THz technologies may be the final link to some fixed, portable AP under the assumption of efficient antenna auto-alignment and dynamic routing algorithms. As discussed, strong obstructions drasti- 


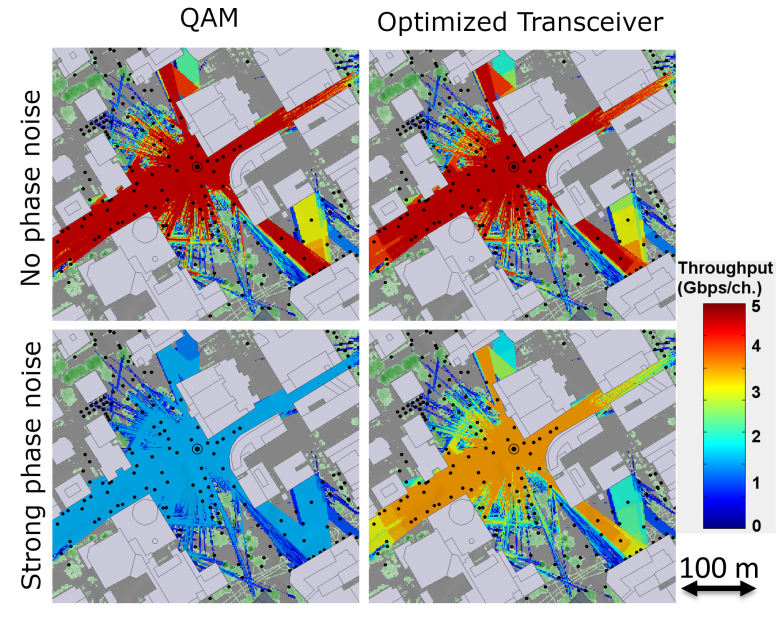

Fig. 1: Comparison of standard and proposed solutions when impaired by PN.

cally impact propagation, thus imply deployment in a clear space.

For the evaluation of the link capacities we assume a link-layer based on LDPC coded P-QAM with a model of commercial hall. Fig. 2 depicts the two-floor $120 \mathrm{~m} \times 80 \mathrm{~m}$ building, which is composed of a large entrance area, large alleys with few obstacles (booths, vegetation, and pillars), and a succession of shops. Each floor is five meters high. A mesh sub-THz network has been designed to feed the wireless AP located at the first floor and entrance area. Two different kinds of AP are considered: those inside the shops, which require a sub- $\mathrm{THz}$ relay to be positioned on the outer surface of the shop window; and those connected to a portable/mobile sub$\mathrm{THz}$ relay installed outside the shops. Following radio link are evaluated: i) node-to-node $(\mathrm{N} 2 \mathrm{~N})$; ii) node to the fixed shop relays at height 4 meters (N2R). The sub-THz system parameters are given in Table I. For this scenario we assume a strong PN. The propagation model is enabling a maximum of 2 reflections and 1 diffraction along each ray path. Reflections on the ceiling or floor surfaces are not allowed as they are likely to be obstructed. Shop windows are assumed as opaque surfaces for considered frequency.

The peak throughput on each radio link is predicted with the antennas aligned on either the direct-path or the strongest propagation path. Fig. 2 depicts the calculated throughput for all mesh connections and the best shop-relay connections. Every mesh node is maximum 2-hop distant from the PoP having a greater than $4 \mathrm{Gbps} / \mathrm{channel}$ peak throughput. All shop relays are attached to the mesh network with more than $2 \mathrm{Gbps} / \mathrm{channel}$ peak throughput. This study has been detailed in [14] and has demonstrated the capability of sub$\mathrm{THz}$ technology to address indoor mesh back-haul scenario.

\section{Example of $D 2 D$}

For this last scenario, we assume a short-range transmission with complexity and energy constrained devices. To meet high throughput while considering simple on-off keying (OOK) modulation as well as non-coherent receiver, spatial multi-

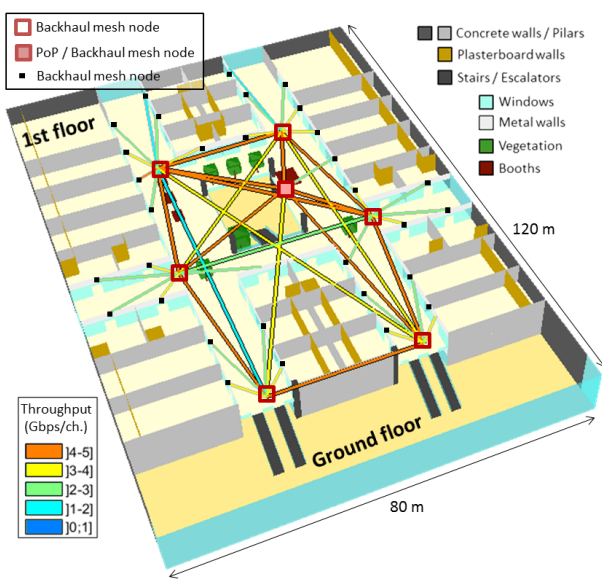

Fig. 2: Connections between mesh nodes, and to the shoprelays.

plexing is investigated as depicted in Fig. 3. In this case each spatial stream is transmitted with a directive antenna. A common challenge is to design a compact system with a low aperture antenna to limit the interference. A recent work has demonstrated that in case of non-coherent demodulation, a trade-off exists between the level of interference between spatial streams (antennas) and the received power when a digital joint demodulation is performed [15].

The main simulation parameters are a carrier frequency of $145 \mathrm{GHz}$, a bandwidth of $2 \mathrm{GHz}$, an antenna gain of $32 \mathrm{dBi}$ with a beam width of $3^{\circ}$ and side lobe level at $-20 \mathrm{dBi}$ and a transmission range of $5 \mathrm{~m}$. Other parameters are assumed identical to the indoor scenario. A uniform linear antennas arrays $N \times N$ MIMO system is considered. The specification of the antenna is extracted from [16] which describes the design of a high-gain antenna for the D-band with transmitarrays and PCB technology.

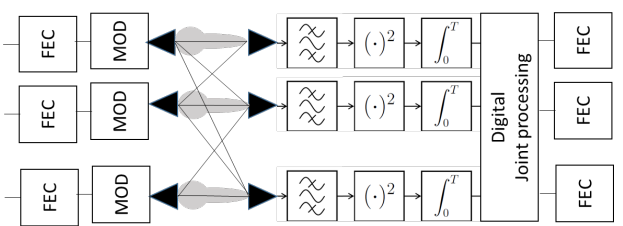

Fig. 3: System model for the D2D scenario.

Further, a polar code scheme is considered to achieve channel coding gain and low packet error rates (PER). The polar code, with a packet size of 512 bytes and a coding rate of $9 / 10$, is decoded with a successive cancellation decoder. The simulated PER with channel coding have been obtained by Monte-Carlo simulations and are reported in Fig. 4 as a function of the level of interference characterized by the following illumination matrix:

$$
\left[\begin{array}{ccccc}
1 & 1 & \rho & \ldots & \rho \\
1 & 1 & 1 & \ddots & \vdots \\
\rho & 1 & 1 & \ddots & \rho \\
\vdots & \ddots & \ddots & \ddots & 1 \\
\rho & \ldots & \rho & 1 & 1
\end{array}\right] .
$$


where $\rho$ models the residual interference due to side lobes of the antennas with $\rho^{2}=-40 \mathrm{~dB}$. We define $\kappa$ as the number of diagonals whose elements are 1 . If $\kappa=1$ then the antennas are almost perfectly spatially multiplexed. Conversely, a large value of $\kappa$ implies that the many channels are strongly correlated.

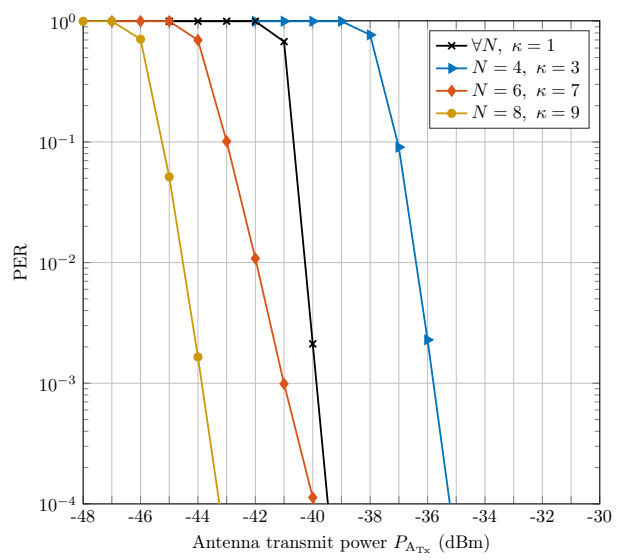

Fig. 4: PER performance for polar coded systems with coding rate $9 / 10$ and OOK modulation scheme

Subsequently, it is shown that an $8 \times 8$ MIMO system may communicate over $5 \mathrm{~m}$ in the D-band with a PER below $10^{-4}$, an antenna transmit power $P_{\mathrm{A}_{\mathrm{Tx}}} \leq-43 \mathrm{dBm}$ and a spatial occupation $\ell \leq 19 \mathrm{~cm}$. The spatial occupation relies on the form factor of the antenna array based on figures from [16]. The data-rate of the system is $7.2 \mathrm{Gbit} / \mathrm{s}$ for a $2 \mathrm{GHz}$ band. A channel bounding scheme, benefiting from the large available sub-THz bands spectrum, could increase the aggregated throughput. It can be concluded that MIMO systems using energy detection receivers may achieve high spectral efficiency wireless communications in sub-THz bands with low power and low complexity RF architectures. Regarding short-range communications, it is worth mentioning a different numerical application where simulation parameters remain the same but $d_{0}=50 \mathrm{~cm}$. It results that a spatial occupation $\ell \leq 1.8 \mathrm{~cm}$ and an antenna transmit power $P_{\mathrm{A}_{\mathrm{Tx}}} \leq-62 \mathrm{dBm}$ ensure a PER below to $10^{-4}$.

\section{E. Summary}

To sum up the requirement and constraints of the three aforementioned scenarios, Table II reports the key performance indicators that will be cross correlated in the next section with available CMOS and III-V technologies. The maximum achievable throughput is given for strong PN and no PN conditions. The transmit power is given per channel and per antenna.

TABLE II: Summary of scenario key performance indicators.

\begin{tabular}{|c|c|c|c|c|c|}
\hline Scenario & $\begin{array}{c}\text { Throughput } \\
\text { no PN - strong PN }\end{array}$ & Channel & $\begin{array}{c}\text { Transmitted } \\
\text { Power }\end{array}$ & Modulation/Coding & Range \\
\hline \hline Outdoor & $4.4-6.2 \mathrm{Gbps} / \mathrm{ch}$ & $1 \mathrm{GHz}$ & $25 \mathrm{dBm}$ & $\begin{array}{c}\text { Coherent P-QAM } \\
\text { LDPC }\end{array}$ & $\sim 300 \mathrm{~m}$ \\
\hline $\begin{array}{c}\text { Indoor } \\
\text { node-to-node }\end{array}$ & $4.4-6.2 \mathrm{Gbps} / \mathrm{ch}$ & $1 \mathrm{GHz}$ & $19 \mathrm{dBm}$ & $\begin{array}{c}\text { Coherent P-QAM } \\
\text { LDPC }\end{array}$ & $\sim 50 \mathrm{~m}$ \\
\hline $\begin{array}{c}\text { Indoor } \\
\text { node-to-relay }\end{array}$ & $2-2.8 \mathrm{Gbps} / \mathrm{ch}$ & $1 \mathrm{GHz}$ & $19 \mathrm{dBm}$ & $\begin{array}{c}\text { Coherent P-QAM } \\
\text { LDPC }\end{array}$ & $\sim 15 \mathrm{~m}$ \\
\hline $\begin{array}{c}\text { D2D } \\
\text { MIMO } 8 \times 8\end{array}$ & $7.2 \mathrm{Gbps} / \mathrm{ch}$ & $2 \mathrm{GHz}$ & $\begin{array}{c}-43 \mathrm{dBm} \\
\text { per antenna }\end{array}$ & $\begin{array}{c}\text { Non-coherent OOK } \\
\text { Polar code }\end{array}$ & $<5 \mathrm{~m}$ \\
\hline
\end{tabular}

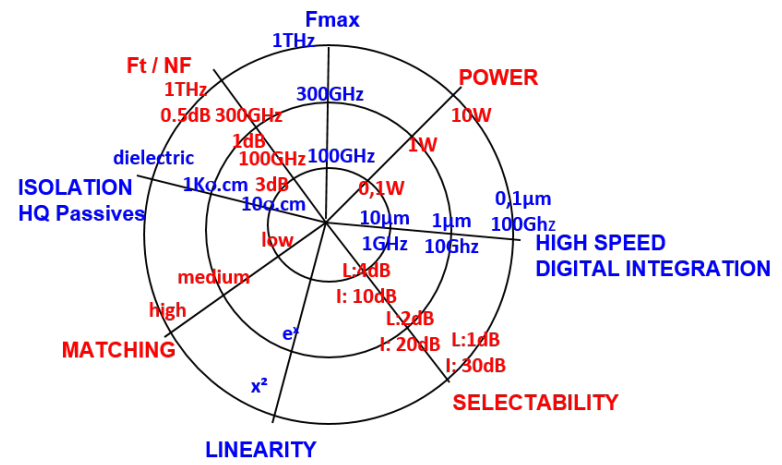

Fig. 5: Process technology targets.

\section{Silicon Based Technologies for Connectivity}

\section{A. Overview and road-maps}

Nowadays, silicon-based technologies offer low cost compromises for RF and mmWaves applications. In this section, their potential and limits are studied for connectivity applications, taking into account connectivity roadmap defined in H2020 NEREID CSA project [17]. For the sake of comparison, process technology characteristics can be considered w.r.t. building blocks performance, such as PA, low noise amplifier (LNA), voltage-controlled oscillator (VCO), and transceivers as depicted in Fig. 5.

Output power ability depends on Bias Voltage (BV) and $I_{\max }$ current. High Speed Digital Integration capability is inversely proportional to the size and efficiency (transit time / current) of transistors. The selectability is the ability to switch RF signals with high isolation and low loss. The linearity of the transistors is given by their output power versus input power relation $\left(x^{2}\right.$ or $\left.e^{x}\right)$. Matching property is considered for two minimum size transistors in a differential structure. Substrate resistivity and Thick Metal play a role on High Quality (HQ) passives limiting pulling effect. The ratio $\mathrm{F}_{t} / \mathrm{NF}$ is a FOM given by the $\mathrm{F}_{t}$ and the $\mathrm{NF}_{\min }$ of the technology $\left(\mathrm{F}_{t}\right.$ for high frequency digital applications and $\mathrm{NF}_{\min }$ for receiver noise). $\mathrm{F}_{\max }$ represents the $G_{p}=0 \mathrm{~dB}$ point, involving applications maximum frequency should be under $\mathrm{F}_{\max } / 3$ for performance achievements. The NEREID connectivity roadmap has also considered the three main application domains, namely D2D, indoor and outdoor applications, and, figures are given for the 5- and 10-year terms (medium- to long-term). Based on this analysis, the connectivity requirements for each process technology characteristics have been extracted and are illustrated in Fig. 6, the main parameter being output power.

\section{B. Position of silicon and III-V technologies}

Silicon-based processes are compared to III-V / InP based HBT ones and FOMs are depicted in Fig. 7. Si-Based FDSOI, InGaAs/Si, B55 and DOT 7, as well as III-V InP offer interesting properties to address the mmWave bands. The benefits of B55, FDSOI and InGaAS/Si are the ability to allow integration of high speed signal processing. Depending on the required output power, B55 and III-V InP have interesting 
capabilities (see e.g [2]). RF-SOI and new GaN/Si bring RF power and selectability.

\section{Case of $P A$}

For each scenarios given in Table II, potential technologies of interest are considered from power amplification side. As the frequency of interest is high w.r.t to $\mathrm{F}_{\max }$, only "A-Class" PA are considered: the operation frequency is less than $\mathrm{F}_{\max } / 3$, but still no current technology is for $5 \times \mathrm{F}_{\max }$ which would made "AB-Class" possible. On the output power side, we have to consider that it is transmitted either isotropically or focusedbeam, this latter requiring a multi-patch antenna, where a single antenna is used for relatively isotropic radiation. Thereby, focused-beam are preferred for mmWave communications, as a group of patches, $4(2 \times 2)$ or $16(4 \times 4)$ [18] namely a tile, is fed by an individual PA, reducing individual PA output power needs. Phased-array or transmit-array architectures are combining individual power into radiated power, built upon phase-shifter on the signal path or primary radiated signal to a transmit-array. Hence, individual PA output power sum is expected as the final radiated power, and as low as $P_{P A}=0$ $\mathrm{dBm}$ to $10 \mathrm{dBm}$ are sufficient. For instance, $25 \mathrm{dBm}$ radiated power can be addressed with $P_{P A}=13 \mathrm{dBm}$ 4-tile array or $P_{P A}=7 \mathrm{dBm}$ 16-tile array, this latter being compatible with silicon technologies, as $8 \mathrm{dBm} / 140 \mathrm{GHz}$ is currently viewed as achievable.

FOMs from an Indoor WiGig application are also given for comparison. It should be mentioned that similar applications are envisaged or already deployed in the V-band. Moreover, it has to be noticed that for D-band the propagation loss penalty is only $7.35 \mathrm{~dB}$ w.r.t V-band and could be negligible or even favorable to D-band for range above $0.5 \mathrm{~km}$. Moreover, benefits of the channel bounding in the D-band clearly show up, as the BW in the V-band is limited by worldwide regulation $(\approx 5 \mathrm{GHz})$.

\section{CONCLUSION}

Competition between technologies, III-V and $\mathrm{Si}$, has been demonstrated as PA output power can be drastically reduced to cover scenarios that have been investigated benefiting from the directivity gain of multiple tiles antenna arrays. Nevertheless, the gain of the PA stage must also be considered as not too many amplification stage are expected to maintain reasonable linearity, cost and consumption. An improvement by a factor 2 for $\mathrm{F}_{t}$, and $\mathrm{F}_{\max }$, would definitively help silicon to become a definitive competitor for Indoor and D2D wireless connectivity

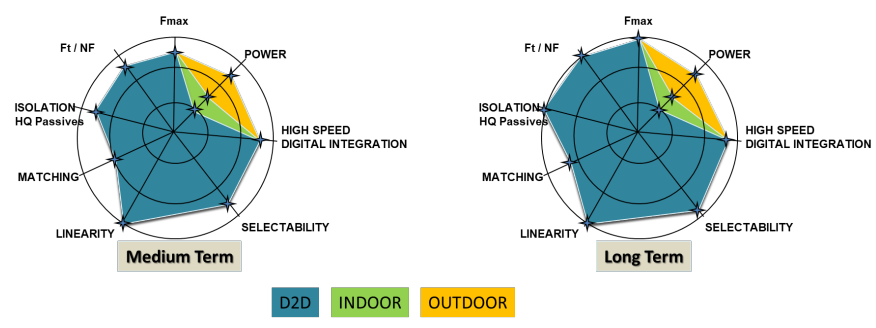

Fig. 6: Connectivity roadmap.
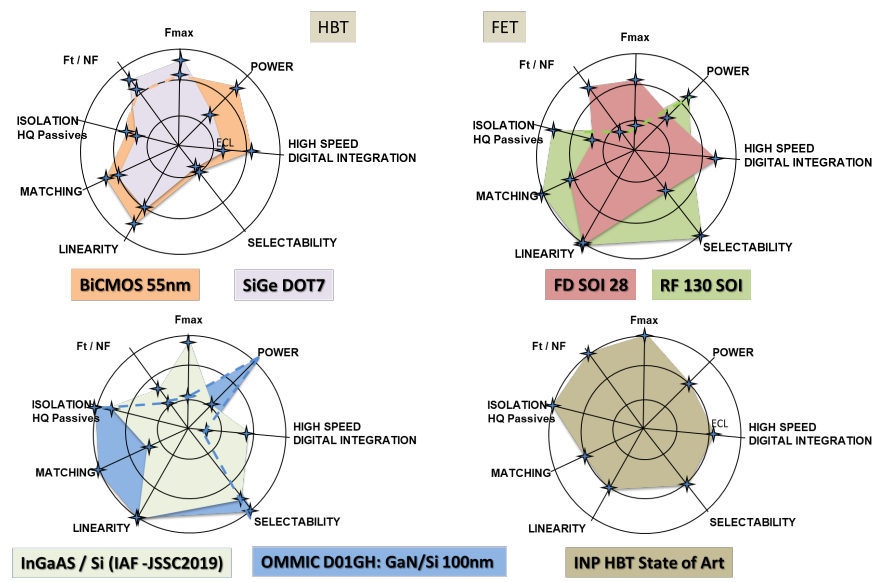

Fig. 7: Position of Si-Based and III-V processes.

[19]. The cost will be the main key factor to earn market shares in these market fields. BiCMOS and RF CMOS processes for Outdoor connectivity could also compete as they can also embed some processing thanks to the better integration they offer. Indeed, on this market, the trend is not to do SoC (System-on-Chip), but rather SiP (System-in-Package). Therefore doors are still open for hybrid integration. RF SOI CMOS and FD-SOI have already proven to be adequate up to $40 \mathrm{GHz}$, providing better integration and overall cost than SiGe or InP HBT, but could also meet the specifications for B5G from mmW to sub- $\mathrm{THz}$ connectivity.

\section{REFERENCES}

[1] E. Calvanese Strinati et al., "6G: The Next Frontier: From Holographic Messaging to Artificial Intelligence Using Subterahertz and Visible Light Communication," IEEE Vehicular Technology Magazine, Sep. 2019.

[2] H. Hamada et al., "Millimeter-wave InP Device Technologies for Ultrahigh Speed Wireless Communications toward Beyond 5G," in IEEE International Electron Devices Meeting, 2019.

[3] J.-B. Doré et al., "Above-90GHz Spectrum and Single-Carrier Waveform as Enablers for Efficient Tbit/s Wireless Communications," in 25th International Conference on Telecommunications , Jun. 2018.

[4] F. Barrera et al., "A D-band 4-ways power splitter and combiner implemented on a 28nm bulk CMOS process," in 2019 49th European Microwave Conference, Oct 2019.

[5] P. Rodríguez-Vázquez et al., "A 16-QAM 100-Gb/s 1-M Wireless Link With an EVM of 17p.c at $230 \mathrm{GHz}$ in an SiGe Technology," IEEE Microwave and Wireless Components Letters, April 2019.

[6] I. Dan et al., "A Superheterodyne $300 \mathrm{GHz}$ Wireless Link for Ultra-Fast Terahertz Communication Systems," in 2019 49th European Microwave Conference, Oct 2019.

[7] S. Bicaïs and J.-B. Doré, "Phase Noise Model Selection for Sub-THz Communications," in 2019 IEEE Global Communication Conference, Dec 2019.

[8] S. Bicaïs et al., " Optimized Single Carrier Transceiver for Future SubTeraHertz Applications ," in International Conference on Acoustics, Speech and Signal Processing, May 2020.

[9] "IEEE Standard for High Data Rate Wireless Multi-Media NetworksAmendment 2: $100 \mathrm{~Gb} / \mathrm{s}$ Wireless Switched Point-to-Point Physical Layer," IEEE Std 802.15.3d-2017 (Amendment to IEEE Std 802.15.32016 as amended by IEEE Std 802.15.3e-2017), pp. 1-55, Oct 2017.

[10] M. Saad et al., "Single carrier with index modulation for low power terabit systems," in 2019 IEEE Wireless Communications and Networking Conference, April 2019.

[11] L. Pometcu and R. D'Errico, "An Indoor Channel Model for High Datarate Communications in D-band," IEEE Access, 2019. 


\begin{tabular}{|c|c|c|c|c|c|c|c|c|c|}
\hline & \multirow[t]{2}{*}{ Frequency } & \multirow[t]{2}{*}{ Throughput } & \multirow[t]{2}{*}{ Channel } & \multirow[t]{2}{*}{$\begin{array}{l}\text { Transmitted } \\
\text { Power }\end{array}$} & \multirow[t]{2}{*}{ Range } & \multicolumn{2}{|c|}{$\begin{array}{l}\text { A Class PA with } \\
\text { Min Fmax }=4356 \mathrm{~Hz} \\
\text { Process to be used }\end{array}$} & \multirow{2}{*}{\multicolumn{2}{|c|}{$\begin{array}{l}\text { Low power signal processing } \\
\text { Min Ft I process }\end{array}$}} \\
\hline & & & & & & One tile & Tile array & & \\
\hline Outdoor & $145 \mathrm{GHz}$ & $4.4-6.2 \mathrm{Gbps} / \mathrm{ch}$ & $1 \mathrm{GHz}$ & $25 \mathrm{dBm}$ & $300 \mathrm{~m}$ & InP HBT & $\begin{array}{l}\quad 16 \text { tiles } \\
\text { Dot } 7 \mathrm{HBT} \\
\text { InGaAs/Si }\end{array}$ & $62 \mathrm{GHz}$ & \multirow{5}{*}{$\begin{array}{c}\text { All CMOS } \\
\text { processes } \\
\text { under } 65 \mathrm{~nm} \\
\text { node }\end{array}$} \\
\hline Indoor node-to-node & $145 \mathrm{GHz}$ & $4.4-6.2 \mathrm{Gbps} / \mathrm{ch}$ & $1 \mathrm{GHz}$ & $19 \mathrm{dBm}$ & $50 \mathrm{~m}$ & $\operatorname{InP}$ HBT & $\begin{array}{l}\quad 4 \text { tiles } \\
\text { Dot } 7 \text { HBT } \\
\text { InGaAs/Si }\end{array}$ & $62 \mathrm{GHz}$ & \\
\hline Indoor node-to-relay & $145 \mathrm{GHz}$ & $2-2.8 \mathrm{Gbps} / \mathrm{ch}$ & $1 \mathrm{GHz}$ & $19 \mathrm{dBm}$ & $15 \mathrm{~m}$ & InP HBT & $\begin{array}{l}\quad 4 \text { tiles } \\
\text { Dot } 7 \mathrm{HBT} \\
\text { InGaAs/Si }\end{array}$ & $28 \mathrm{GHz}$ & \\
\hline $\begin{array}{c}\text { D2D } \\
\text { MIMO } 8 \times 8\end{array}$ & $145 \mathrm{GHz}$ & $7.2 \mathrm{Gbps} / \mathrm{ch}$ & $2 \mathrm{GHz}$ & $\begin{array}{c}-43 \mathrm{dBm} \\
\text { per antenna }\end{array}$ & $5 \mathrm{~m}$ & $\begin{array}{l}\text { InP HBT } \\
\text { Dot } 7 \text { HBT } \\
\text { InGaAs/Si }\end{array}$ & 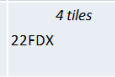 & $72 \mathrm{GHz}$ & \\
\hline WiGig indoor & $66 \mathrm{GHz}$ & $4.6 \mathrm{Gbps} / \mathrm{ch}$ & $2 \mathrm{GHz}$ & $10 \mathrm{dBm}$ & $10 \mathrm{~m}$ & $\begin{array}{l}\text { InGaAs/Si } \\
\text { FDSOI } \\
\text { Dot } 7 \text { HBT }\end{array}$ & Not required & $62 \mathrm{GHz}$ & \\
\hline
\end{tabular}

TABLE III: Technologies candidates for PA.

[12] T. Xing and T. S. Rappaport, "Propagation Measurement System and Approach at $140 \mathrm{GHz}-$ Moving to 6G and Above $100 \mathrm{GHz}$," in 2018 IEEE Global Communications Conference (GLOBECOM), Dec 2018.

[13] G. Gougeon, Y. Corre, and M. Z. Aslam, "Ray-based Deterministic Channel Modelling for sub-THz Band," in 2019 IEEE International Symposium on Personal, Indoor and Mobile Radio Communications, Sept 2019.

[14] G. Gougeon et al., "Assessment of sub-THz Mesh Backhaul Capabilities from Realistic Modelling at the PHY Layer," in European Conference on Antennas and Propagation, March 2020.

[15] S. Bicaïs, J.-B. Doré, and V. Savin, "Design of MIMO Systems with Energy Detection Receivers for Future Sub-TeraHertz Applications," in under submission, 2020.

[16] F. F. Manzillo, A. Clemente, and J. L. Gonzalez-Jiménez, "High-gain D-band Transmitarrays in Standard PCB Technology for Beyond-5G Communications," IEEE Transactions on Antennas and Propagation, 2019.

[17] NEREID H2020, "Deliverable 8.3: Technology Outlook in Nanoelectronics and harmonization of NEREID roadmaps with International Roadmaps," Tech. Rep., 2008.

[18] S. Shahramian and M. Holyoak, "The Limits of Antenna and Interconnect - Integration for Large-Scale Phased Arrays," in WSC-8, International Microwave Symposium 2019.

[19] T. V. Dinh et al., "Record High-performance RF Devices in an Advanced FDSOI Process Enabling Integrated Watt-level Power Amplifiers for WiFi and 5G applications," in IEEE International Electron Devices Meeting, 2019. 